\title{
Universality of ordering dynamics in conserved multicomponent systems
}

\author{
Jeppesen, Claus; Mouritsen, Ole G.
}

Published in:

Physical Review B

Link to article, DOI:

10.1103/PhysRevB.47.14724

Publication date:

1993

Document Version

Publisher's PDF, also known as Version of record

Link back to DTU Orbit

Citation (APA):

Jeppesen, C., \& Mouritsen, O. G. (1993). Universality of ordering dynamics in conserved multicomponent systems. Physical Review B, 47(22), 14724-14733. https://doi.org/10.1103/PhysRevB.47.14724

\section{General rights}

Copyright and moral rights for the publications made accessible in the public portal are retained by the authors and/or other copyright owners and it is a condition of accessing publications that users recognise and abide by the legal requirements associated with these rights.

- Users may download and print one copy of any publication from the public portal for the purpose of private study or research.

- You may not further distribute the material or use it for any profit-making activity or commercial gain

- You may freely distribute the URL identifying the publication in the public portal

If you believe that this document breaches copyright please contact us providing details, and we will remove access to the work immediately and investigate your claim 


\title{
Universality of ordering dynamics in conserved multicomponent systems
}

\author{
Claus Jeppesen \\ Department of Physical Chemistry, The Technical University of Denmark, Building 206, DK-2800 Lyngby, Denmark \\ and Department of Physics, University of California, Los Angeles, California 90024 \\ Ole G. Mouritsen \\ Department of Physical Chemistry, The Technical University of Denmark, Building 206, DK-2800 Lyngby, Denmark
}

(Received 27 October 1992)

\begin{abstract}
A comparative study is performed of the ordering dynamics and spinodal decomposition processes in two-dimensional two-state and three-state ferromagnetic Potts models with conserved order parameter. The models are investigated by Monte Carlo quenching simulations on both square and triangular lattices and the evolving order is studied via the excess energy, the domain-size distribution function, and the density of isolated diffusing particles, which facilitate the coarsening process. The growth law that describes the time-evolution of the linear length scale, $R(t)$, of the ordered domains is found at late stages to be algebraic, $R(t) \sim t^{n}$, with the Lifshitz-Slyozov value of the exponent, $n \simeq \frac{1}{3}$, for both twoand three-component order parameters. The domain-size distribution function is found to obey dynamical scaling. The results suggest that, similar to the case of nonconserved order parameter, there is a single universality class describing the cases of conserved order parameter independent of the number of components of the order parameter. In the asymptotic regime, the topological difference in the domainboundary network between the vertex-free two-state model and the vertex-generating three-state model does not affect the growth exponents but only the nonuniversal amplitudes. Details are revealed of the ordering mechanism controlled by diffusional processes involving broken Potts bonds and isolated Potts spins. A transient regime can be identified as one where broken Potts bonds in the two-state model and broken Potts bonds (isolated Potts) spins in the three-state model diffuse along the domain boundaries and an asymptotic late-stage regime where isolated Potts spins perform a long-range diffusive process within and across the domains.
\end{abstract}

\section{INTRODUCTION}

Spinodal decomposition ${ }^{1-3}$ is the process displayed by a system that phase separates after having been quenched into an unstable state by a sudden change in thermodynamic variables, such as temperature or chemical potential. After the quench, the system is unstable to longwavelength fluctuations, there is no nucleation barrier, and the system coarsens at late stages by an evaporationcondensation process, as described by the classical theory due to Lifshitz and Slyozov.,4 During the spinodaldecomposition process, the system develops order in different domains corresponding to the various components of the order parameter. The domains are separated by a domain-boundary network carrying the nonequilibrium excess free energy, which provides the driving forces for the separation process. It is the curvature of the boundaries which controls the growth of the domains. The late stage of the growth is governed by long-range diffusion of material across the domains. According to the Lifshitz-Slyozov theory, the timedependent linear length scale of the growing domains exhibits an algebraic growth law

$$
R(t) \sim t^{1 / 3},
$$

i.e., with a growth exponent of value $n=\frac{1}{3}$.

Spinodal decomposition and the applicability of the
Lifshitz-Slyozov growth mechanism are usually associated with cases where the order parameter of the system is a conserved quantity. This need not be the case, however, since the only requirement is that long-range diffusional processes control the asymptotic coarsening process. For many systems, e.g., fluid mixtures, this requirement automatically implies that the order parameter is conserved. However, in systems where the domains have a superstructure and separate into phases of different density, ${ }^{5-9}$ long-range diffusion is needed to facilitate the ordering process, and the Lifshitz-Slyozov growth law should apply even though the order parameter is not conserved. More complicated cases arise when nonconserved order parameters are coupled to conserved modes. ${ }^{3,10}$ In the present work, we shall consider spinodal decomposition processes for a conserved order parameter which is not coupled to other modes.

The standard case of spinodal decomposition ${ }^{3}$ considers a system with two order-parameter components $(p=2)$, e.g., a binary fluid mixture or a binary alloy. In this case, by simple topological arguments, the domain boundaries are $(d-1)$-dimensional surfaces and no vertices can form. The question arises as to the effect of vertex formation on the growth dynamics in the case of $p>2$, e.g., for a ternary alloy. We shall address the question in the present paper by performing a comparative study of two-dimensional model systems undergoing spinodal decomposition and phase separation in the cases of 
both a two- and a three-component conserved order parameter. We provide evidence from computer-simulation calculations on the two-dimensional two-state $(p=2)$ and the two-dimensional three-state $(p=3)$ ferromagnetic Potts models with conserved order parameter that the number of components is irrelevant for determining the asymptotic growth exponent.

The question of the influence of the number of components is of fundamental interest in relation to a possible universal classification of systems undergoing nonequilibrium ordering processes. It is now well established in the case of a nonconserved order parameter, ${ }^{3,11}$ where the domain growth is controlled by short-range diffusion processes, that the late-stage ordering process is described by the classical Lifshitz-Allen-Cahn ${ }^{3}$ growth law

$$
R(t) \sim t^{1 / 2},
$$

i.e., with a growth exponent of value $n=\frac{1}{2}$, independent of spatial dimension, material parameters, details of the interactions, and the number of components of the order parameter. Hence the universality class for nonconserved order parameters is very large. The case of conserved order parameters is much more controversial, although it now appears from both experiment, ${ }^{2,12-17}$ theory, ${ }^{1-4,10,18-28}$ and numerical work ${ }^{21,29-51}$ that the growth law in Eq. (1) applies independent of spatial dimension, material parameters, and details of the interaction potentials. It still remains to be investigated what the effect of the number of order-parameter components may be.

One reason why the case of conserved order parameter is more difficult to resolve as far as the late-stage ordering dynamics is concerned is that the growth is hampered by intermediate-stage transient effects which slow down the ordering process. A dominant transient effect is caused by growth via diffusion along the domain-boundary network which can be shown to lead to another algebraic growth $\operatorname{law}^{6,21}$

$$
R(t) \sim t^{1 / 4}
$$

with a rather low growth exponent $n=\frac{1}{4}$. In the present paper, we are also going to investigate this transient behavior, which turns out to be more dominant the more order-parameter components are involved in the ordering process. By studying the formation of kinks at the domain boundaries and the density of material diffusing inside the domains, respectively, we provide some insight into the microscopic phenomena controlling the growth.

Recent theoretical work on the dynamics of spinodal decomposition has mainly focused on systems with two components. A number of field-theoretical, analytical calculations $3,23,26,27$ including renormalization-group calculations $^{22,24}$ have been presented. The numerical work is based either on numerical solutions to phenomenological models, such as the Langevin and Cahn-Hilliard equations, ${ }^{29,31-34,36,37,41}$ nonlinear coupled-map equations, ${ }^{30,40}$ Monte Carlo renormalization-group calculations, ${ }^{42,43}$ or conventional Monte Carlo simulations on kinetic lattice models. ${ }^{21,44-49,51}$ Some of the problems involved in dynamics of ordering in conserved multicom- ponent systems were addressed in an early computersimulation study by Grest and Sahni, ${ }^{44}$ who found very low growth exponents $n \simeq 0.2$ in the $p=3$ and $p=6$ state conserved Potts model. As we shall discuss in the present paper, these findings are related to transient effects.

In Sec. II we describe the simulation methods that we have applied to investigate the spinodal decomposition processes in the two- and three-state Potts models. This section also includes a brief discussion of choices of time parameters in stochastic models of ordering dynamics. The results for the ordering dynamics are described in Sec. III in terms of microstructure evolution, growth laws, and dynamical scaling. A brief account of some of this work was published in Ref. 51. This section also contains some detailed results related to the microscopic growth mechanism. The results obtained are summarized and discussed in the context of universality in Sec. IV.

\section{MODEL AND METHOD}

\section{A. Potts model}

The ferromagnetic Potts model is defined by the Hamiltonian

$$
H=-J \sum_{\langle i, j\rangle} \delta_{\sigma_{i}, \sigma_{j}},
$$

with $J>0$, and the summation is extended over nearestneighbor pairs on either a square or a triangular lattice. The Potts spin variable $\sigma_{i}$ takes on the values 1,2 for the two-state model (the Ising model) and 1,2,3 for the threestate model. The lattices are subject to periodic boundary conditions, and two different lattice sizes $N=L \times L$ have been considered corresponding to $L=200$ and 400 . The bulk of the statistical analysis has been performed on simulation data obtained from the lattice with $200 \times 200$ sites. The critical temperatures are $J / k_{B} T_{c}=0.8813736$ and 0.5493008 for the two-state model on the square and triangular lattices, respectively, ${ }^{52}$ and $J / k_{B} T_{c}=1.00505$ and 0.6309447 for the three-state Potts model on the square and triangular lattices, respectively. ${ }^{53,54}$

\section{B. Quenching simulations and measures of growth}

For both the two- and three-state models, simulations have been performed corresponding to quenches from infinite temperature and completely disordered states to a temperature $T=T_{c} / 2$ well within the ordered phase. The microscopic dynamics is that of Kawasaki nearestneighbor pair exchange, which assures that the order parameter is conserved both locally as well as globally. It is important for the spinodal-decomposition problem that the order parameter be conserved locally. ${ }^{49}$ All quenches are done at critical conditions in the sense that the order parameter is zero; i.e., the different Potts states are equally populated. In order to obtain ensemble averages, a large number of independent quenches (typically 30-100) have been performed and used to derive averages.

The nonequilibrium growth phenomena following the thermal quenches are monitored as a function of time by a number of measures. First, we have recorded typical 
microconfigurations to reveal how the domain-boundary network is formed and how it coarsens. Second, we have calculated the total nonequilibrium excess internal energy bound in the entire boundary network and from this quantity derived, via the Binder-Stauffer ${ }^{55}$ scaling assumption, a linear length scale

$$
R_{E}(t)=N\left[\langle\mathscr{H}(t)\rangle-\langle\mathscr{H}\rangle_{T}\right]^{-1} .
$$

The equilibrium energies $\langle\mathscr{H}\rangle_{T}$ needed to calculate $R_{E}(t)$ have been obtained from exact analytical expressions in the case of the two-state model $^{53,54}$ and have been derived by accurate equilibrium simulations in the case of the three-state model.

Third, for the three-state model we have calculated the time-dependent domain-size (area) distribution $P(A, t)$, where $A$ is the area of a domain of the same Potts order. From $P(A, t)$ another time-dependent length scale can be determined,

$$
\begin{aligned}
R_{A}(t) & =[\overline{A(t)}]^{1 / 2} \\
& =\left[\int_{A_{0}}^{\infty} A(t) P(A, t) d A\right]^{1 / 2},
\end{aligned}
$$

which is a measure of the average linear extension of a domain. In Eq. (6) we have introduced a lower cutoff, which we shall return to later. We have not used $R_{A}(t)$ as a length-scale measure for the two-state model since in that case it is less reliable because of the very convoluted and percolative structure of the growing domains. Even in the case of the three-state model, we find that the domain pattern is somewhat convoluted and $R_{A}(t)$ is a less reliable length-scale measure than $R_{E}(t)$.

\section{Relations between time scales}

The microscopic dynamics underlying the ordering process is Kawasaki pair-exchange dynamics. The natural time scale for the associated master equation is that corresponding to attempted pair exchanges per lattice site [Monte Carlo steps per site (MCS/S)]. Since only exchanges of pairs on sites occupied by Potts spins in different states can alter the configuration, we have devised an algorithm which selectively excites these broken bonds. The time scale corresponding to selective excitation of broken bonds (MCBB/S) is different from that of $\mathrm{MCS} / \mathrm{S}$ and is a function of $\mathrm{MCS} / \mathrm{S}$, which reflects the ordering dynamics. Obviously, the number of broken bonds decreases as the ordering process evolves. The relation between the two different time scales is determined in the simulation, and it is used to express the measures of growth in terms of the natural time scale MCS/S. Provided that the list of broken bonds is effectively constructed and updated during the simulation, it is possible to speed up the simulation in a way which is competitive with both the continuous-time method ( $n$-fold way), which has been used to study domain growth in nonconserved multistate Potts models ${ }^{56}$ and with the use of vectorized dynamics. ${ }^{45,47,48,57}$

In Fig. 1 is shown the relation between the two time scales MCS/S and MCBB/S for the two- and three-state models on the square lattice. The relation is extremely

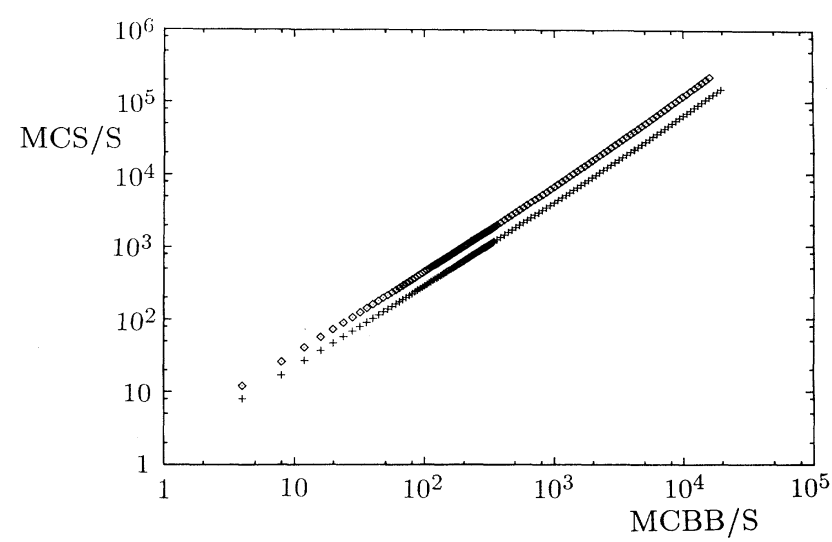

FIG. 1. Log-log plot of the relation between the two time scales MCS/S and MCBB/S involved in simulation of the twostate $(\diamond)$ and three-state $(+)$ Potts models on the square lattice after a quench from infinite temperature to a temperature $T=\frac{1}{2} T_{c}$ below the equilibrium ordering temperature $T_{c}$.

smooth with a slight but systematic deviation from a power-law relation. A similar relationship between different time scales was recently discussed in a simulation study of vacancy-mediated continuous ordering in Ising models with nonconserved order parameter. ${ }^{58}$ The relationship in Fig. 1 is not expected to be algebraic because the number of broken bonds is not simply proportional to the total length of the domain boundary [which is inversely proportional to the length scale $R_{E}(t)$ for sharp domain walls], but also contains a term from the isolated Potts spins diffusing within he domains. We shall return to this point in Sec. III D.

\section{RESULTS}

\section{A. Evolving domain structures}

In Figs. 2 and 3 are shown time series of typical microconfigurations for both the two- and three-state models on the square as well as the triangular lattice. For the three-state model, only the domain-boundary network is shown. From these snapshots the following qualitative observations can be made. First, the domain pattern is very convoluted for the two-state model on both lattices. For the three-state model, the domains are considerably more compact and the domain-boundary network is characterized by vertices where domains of three different types meet. Second, an increasing number of isolated Potts spins within the domains is observed for the two-state model at late stages. For the three-state model, isolated Potts spins appear both along the domain boundaries and within the domains. We shall in Sec. III D return to these observations.

\section{B. Growth laws and growth exponents}

The data for the time dependence of the two length scales $R_{E}(t)$ and $R_{A}(t)$ in Eqs. (5) and (6) for the two models are shown in Figs. 4-6. For reasons described in 
(a)

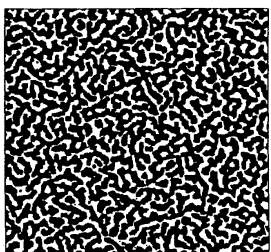

$t=13000$

(b)

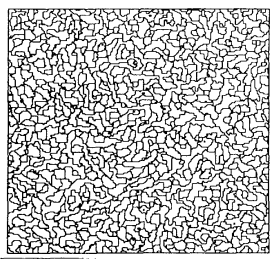

$t=8000$

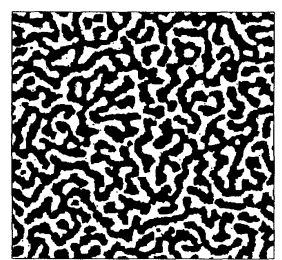

$t=71000$

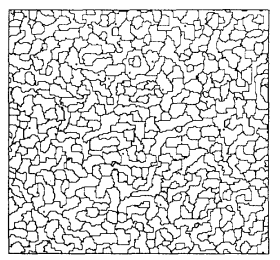

$t=39000$

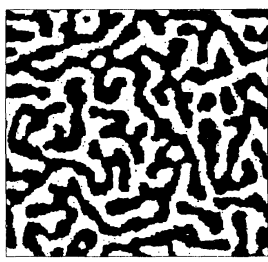

$t=600000$

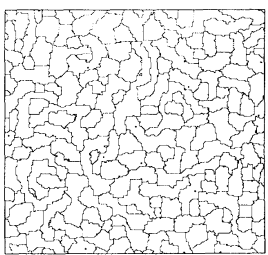

$t=209000$

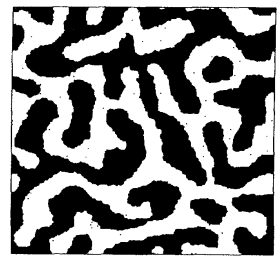

$t=2100000$

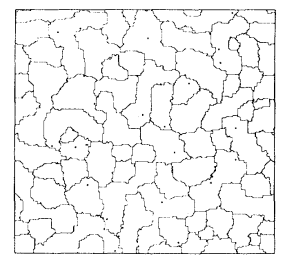

$t=1200000$
FIG. 2. Time evolution of the domain-boundary network for the (a) two-state and the (b) three-state Potts models on a square lattice after a quench from infinite temperature to a temperature $T=\frac{1}{2} T_{c}$ below the equilibrium ordering temperature $T_{c}$. The time $t$ is given in units of $\mathrm{MCS} / \mathrm{S}$. The simulations have been carried out on lattices with $400 \times 400$ sites for the two-state model and $200 \times 200$ sites for the three-state model.
Sec. II, data for $R_{A}(t)$ have only been recorded for the three-state model. In accordance with the evolving microstructures displayed in Figs. 2 and 3, the growth data in Figs. 4-6 show that the overall growth rate is much slower in the three-state model than in the two-state model and that the growth for either model is faster on the square than on the triangular lattice. For calculation of $R_{A}(t)$ in Fig. 6, the lower cutoff in domain size [cf. Eq. (6)] has been chosen to be $A_{0}=6$ Potts spins. The choice of the cutoff is immaterial for the late-stage behavior.

For all the data presented in Figs. 4-6, it appears that in none of these cases is the growth described by a simple power law in any extended time range. All of the curves display a distinct curvature, indicating an increasing value of the effective exponent

$$
n_{\mathrm{eff}}(t)=\frac{d[\ln R(t)]}{d[\ln t]}
$$

Huse $^{21}$ has suggested a simple procedure for extracting the asymptotic value of the growth exponent $n$ from finite-time data for spinodal-decomposition processes. This procedure is built on the assumption that the finitetime effective exponent is an implicit function of time via the time-dependent length scale and is given by

$$
n_{\mathrm{eff}}(t)=n-\frac{a}{R(t)}-\frac{b}{R^{2}(t)}+O\left[R^{-3}(t)\right],
$$

where we have extended Huse's formula to second order. ${ }^{51}$ The first correction term in Eq. (8) takes account of the effects of diffusion along the domain-boundary network. It has been pointed out by Yeung ${ }^{59}$ that the Huse formula should be considered as only an empirical relation and that at present there is no known physical justification for its form. However, Yeung ${ }^{59}$ points out that as an empirical relation it is very powerful and that it gives results in good agreement with theoretical predic-

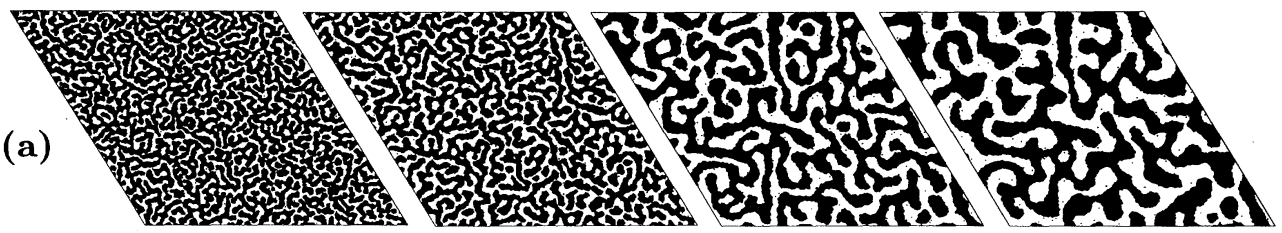

$$
t=2000 \quad t=6900 \quad t=90000 \quad t=280000
$$

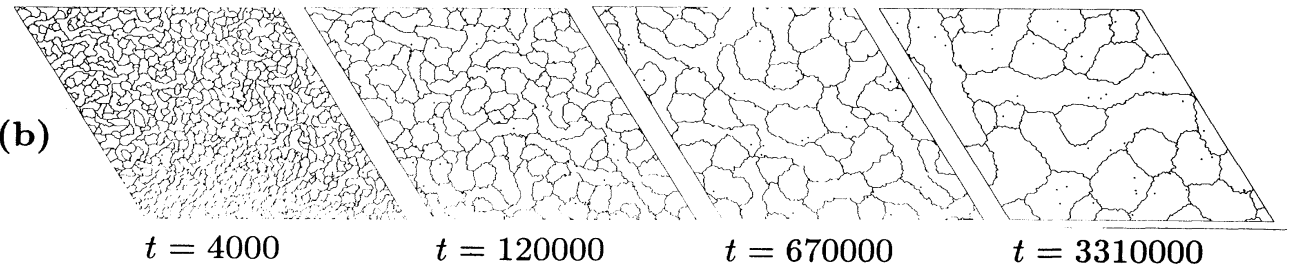

FIG. 3. Time evolution of the domain-boundary network for the (a) two-state and the (b) three-state Potts models on a triangular lattice after a quench from infinite temperature to a temperature $T=\frac{1}{2} T_{c}$ below the equilibrium ordering temperature $T_{c}$. The time $t$ is given in units of MCS/S. The simulations have been carried out on lattices with $400 \times 400$ sites for the two-state model and $200 \times 200$ sites for the three-state model. 


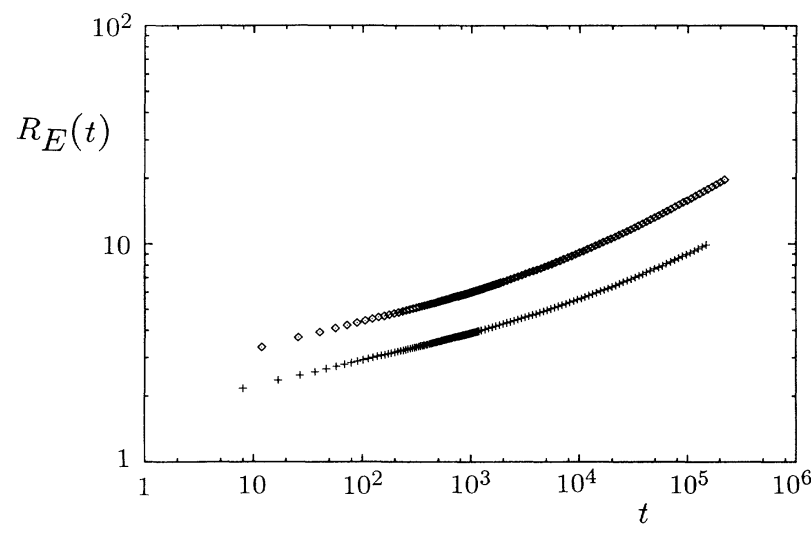

FIG. 4. Log-log plot of the time dependence of the inverse excess energy, $R_{E}(t)$ in Eq. (5), for the two-state $(\diamond)$ and the three-state $(+)$ Potts models on the square lattice after a quench from infinite temperature to a temperature $T=\frac{1}{2} T_{c}$ below the equilibrium ordering temperature $T_{c}$. The time is in units of Monte Carlo steps per site.

tions for spinodal-decomposition processes in twocomponent systems. In fact, the first very strong numerical evidence for the validity of the Lifshitz-Slyozov law in the spinodal-decomposition process in the conserved Ising model ${ }^{45,47}$ was based on Huse's formula with $b=0$. Subsequent numerical work on other models with conserved under parameter ${ }^{31,37,48,51}$ also made successful use of the formula.

In the present work, we have applied the extended Huse formula to analyze the growth data in Figs. 4-6 in an attempt to extract the asymptotic growth exponent. The results of this analysis are given in Figs. 7-9.

First, we discuss the results obtained on the basis of the length scale $R_{E}(t)$. It appears that, in the case of the

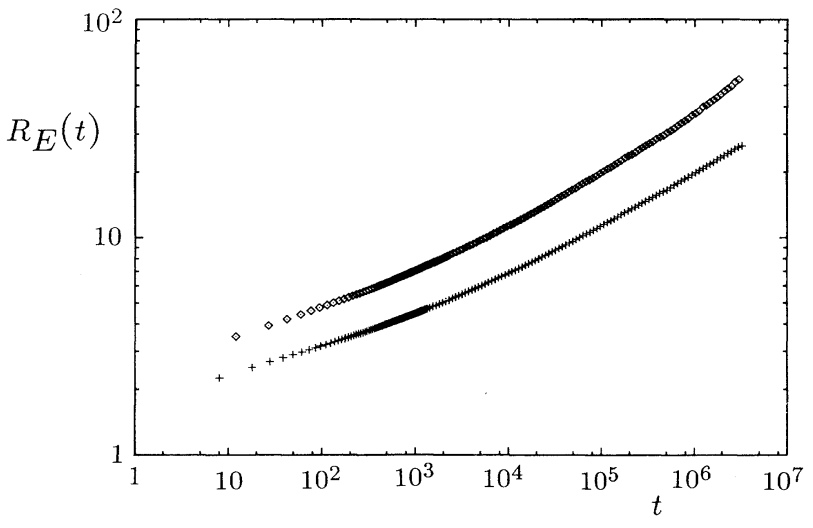

FIG. 5. Log-log plot of the time dependence of the inverse excess energy, $R_{E}(t)$ in Eq. (5), for the two-state $(\diamond)$ and the three-state $(+)$ Potts models on the triangular lattice after a quench from infinite temperature to a temperature $T=\frac{1}{2} T_{c}$ below the equilibrium ordering temperature $T_{c}$. The time is in units of Monte Carlo steps per site.

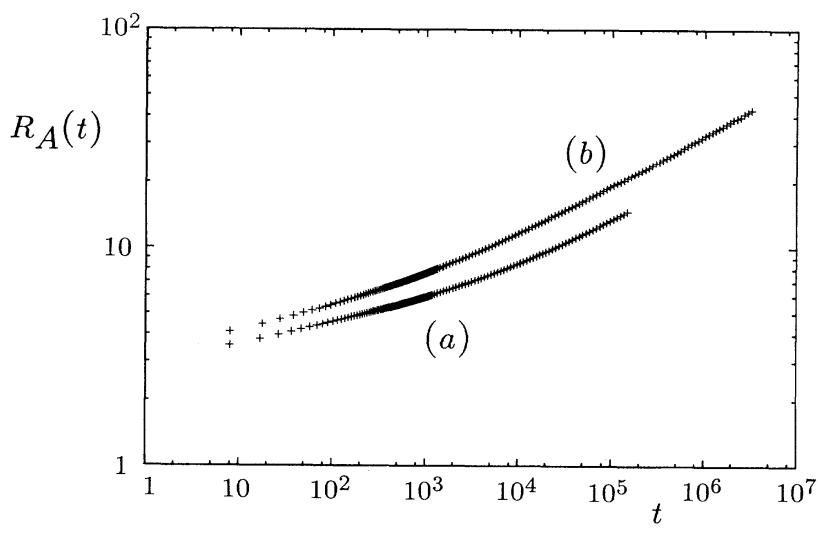

FIG. 6. Log-log plot of the time dependence of the average linear domain size, $R_{A}(t)$ in Eq. (6), for the three-state Potts model on (a) the square lattice and (b) the triangular lattice after a quench from infinite temperature to a temperature $T=\frac{1}{2} T_{c}$ below the equilibrium ordering temperature $T_{c}$. The time is in units of Monte Carlo steps per site.

two-state model on the square lattice, the data for $n_{\text {eff }}$ is consistent with Eq. (8) with $b \simeq 0$. A fit to Eq. (8) with $b=0$ leads to $n \simeq 0.328 \pm 0.005(a=1.082)$, which is consistent with the result obtained by Amar, Sullivan, and Mountain, ${ }^{45} n \simeq 0.330 \pm 0.005$, for the Ising model, on the square lattice. In the case of the three-state model on the square lattice, the data for $n_{\text {eff }}$ in Fig. 7 exhibit a considerable degree of curvature and the second-order term in Eq. (8) has to be taken into account. A fit to the data including both the first- and second-order terms leads to a value $n \simeq 0.344 \pm 0.010(a=1.18$ and $b=1.36)$. We then turn to the data for the triangular lattice (Fig. 8), which appears to approach the asymptotic limit much more slowly than for the square lattice. Similar to the square

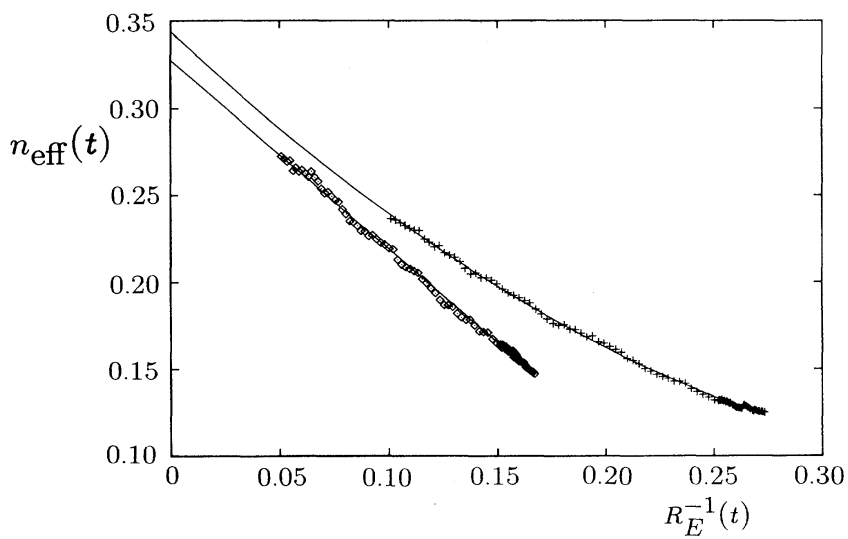

FIG. 7. Effective exponent $n_{\text {eff }}$ in Eq. (7) as a function of inverse length scale, $R(t)=R_{E}(t)$, as shown in Fig. 4 for the twostate $(\diamond)$ and three-state $(+)$ Potts models on the square lattice. The extrapolations (solid lines) to the large-length-scale limit are obtained by fitting the data to the extended Huse formula in Eq. (8). 


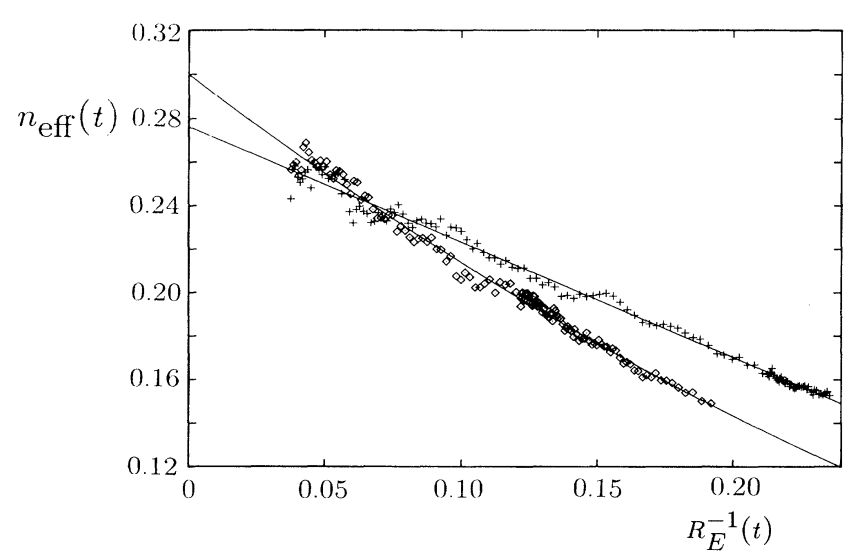

FIG. 8. Effective exponent $n_{\text {eff }}$ in Eq. (7) as a function of inverse length scale, $R(t)=R_{E}(t)$, as shown in Fig. 5 for the twostate $(\diamond)$ and three-state $(+)$ Potts models on the triangular lattice. The extrapolations (solid lines) to the large-length-scale limit are obtained by fitting the data to the extended Huse formula in Eq. (8).

lattice, it is found that the three-state model approaches the asymptotic growth behavior slower than the two-state model. An analysis based on the extended Huse formula [Eq. (8)] for the growth data for the triangular lattice leads to $n \simeq 0.30+00.01(a=0.94$ and $b=0.79)$ for the two-state model and $n \simeq 0.28 \pm 0.01 \quad(a=0.53$ and $b=0.003$ ) for the three-state model.

To determine the asymptotic value of the growth exponent using the growth data for the length scale $R_{A}(t)$ in Fig. 6 is not so straightforward. The reason for this is that in the finite-time regime, which we can access in our simulations, the properties derived from the domain-size distribution function depend on the value chosen for the lower cutoff, $A_{0}$ in Eq. (6). There are two reasons why it is necessary to introduce such a cutoff in the analysis of

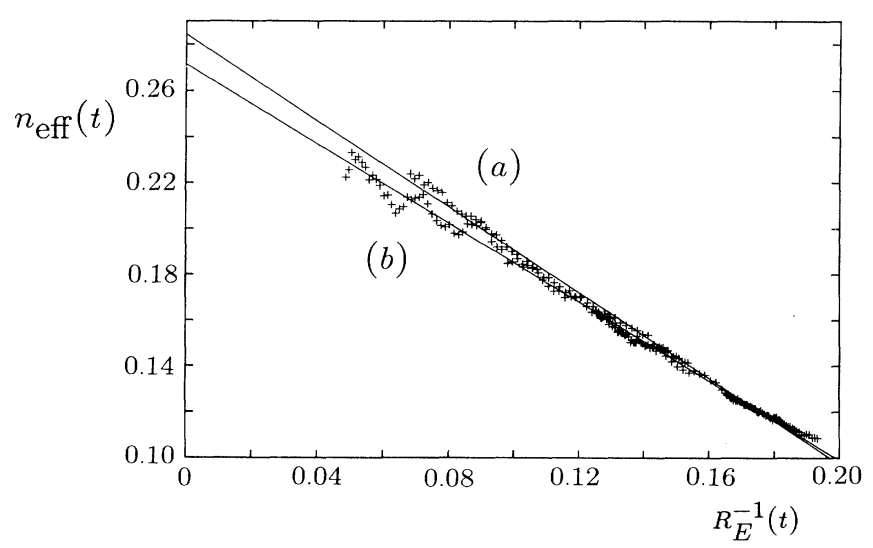

FIG. 9. Effective exponent $n_{\text {eff }}$ in Eq. (7) as a function of inverse length scale, $R(t)=R_{A}(t)$, as shown in Fig. 6 for the three-state Potts model on (a) the square lattice and (b) the triangular lattice. The extrapolations (solid lines) to the largelength-scale limit are obtained by fitting the data to the Huse formula in Eq. (8). the data of finite lattices at finite times. First, the isolated Potts spins ( $A=1$ ) have to be excluded. These isolated spins cannot be considered as domains. Second, domains of an area smaller or equal to the area covered by a connected string of Potts spins adsorbed in a typical interface between two domains should be excluded. This area is proportional to $R_{A}(t)$. It is clear from the microconfigurations in Figs. 2(b) and 3(b) that such "domains" occur and that they cannot be considered as ordered domains. Since this latter cutoff itself is a function of the cutoff, we have decided to choose somewhat arbitrarily a value of $A_{0}=6$, which assures that the smallest "nondomains" are excluded from the averaging procedure. Figure 9 presents the data for $n_{\text {eff }}$ as a function of $R_{A}(t)$ for this value of the cutoff. From a fit to the data of the Huse formula in Eq. (8) (where we have fixed $b=0$ because of the large scatter in the data), we find for the three-state model $n \simeq 0.28 \pm 0.01 \quad(a=0.94)$ for the square lattice and $n \simeq 0.27 \pm 0.01(a=0.86)$ for the triangular lattice. For larger values of the cutoff, the extrapolated values of the asymptotic exponents will be larger. Because of the particular difficulties mentioned in Sec. II B in using $R_{A}(t)$ as a length-scale measure for systems with very convoluted domain structures and because of the large scatter in the data in Fig. 9, we attach less confidence to the growth-exponent values quoted above for $R_{A}(t)$. They are likely not to be the true asymptotic values.

\section{Dynamical scaling}

We shall now analyze the spinodal decomposition processes in the three-state model in terms of dynamical scaling. We use the data for the time-dependent domain-size (area) distribution function $P(A, t)$. Dynamical scaling of the domain-size distribution function implies the existence of a scaling function

$$
\widetilde{P}(x)=\overline{A(t)} P(A, t),
$$

where the scaling variable is $x=A(t) / \overline{A(t)}$. In Figs. 10

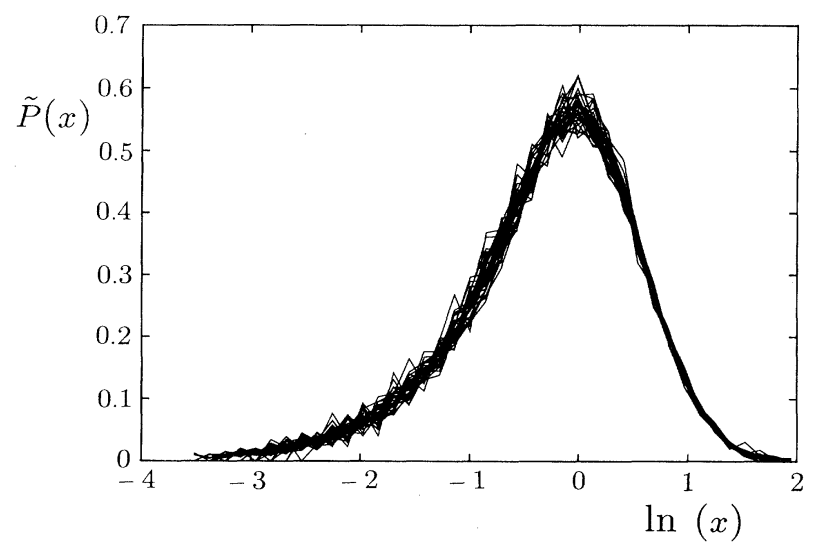

FIG. 10. Scaling function $\widetilde{P}(x)$ in Eq. (9) for the domain-size distribution function of the three-state Potts model on the square lattice, where the scaling variable is $x=A(t) / \overline{A(t)}$. The data cover the time range $t=10000-200000 \mathrm{MCS} / \mathrm{S}$. 


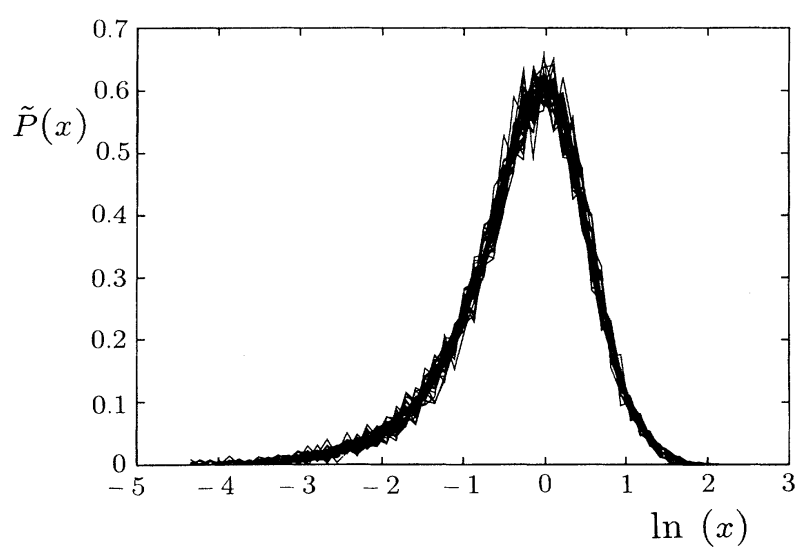

FIG. 11. Scaling function $\widetilde{P}(x)$ in Eq. (9) for the domain-size distribution function of the three-state Potts model on the triangular lattice, where the scaling variable is $x=A(t) / \overline{A(t)}$. The data cover the time range $t=10000-200000 \mathrm{MCS} / \mathrm{S}$.

and 11 , the data for $P(R, t)$ are plotted using Eq. (9). It is seen that for both lattices the data for $P(R, t)$ at late times can be collapsed into a single function. Hence the spinodal-decomposition process for the three-component systems obeys dynamical scaling at late stages. It is noted that scaling seems to be obeyed by our data despite the fact that the asymptotic growth regime has not been attained in terms of the simple algebraic growth law [Eq. (1)]. In Fig. 12 the scaling functions for the two lattices are compared. The data used in this figure are obtained from Figs. 10 and 11 by averaging over the entire data set in the scaling regime. It appears that there is only little, if any, difference between the scaling functions for the two lattices.

\section{Details of the growth mechanism}

Contrary to the approach to spinodal-decomposition processes which is based on phenomenogical field equa-

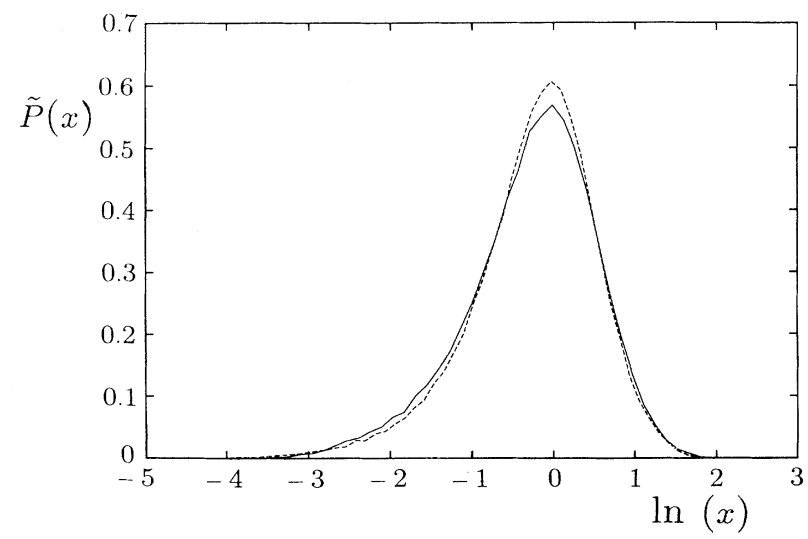

FIG. 12. Comparison of the dynamical scaling functions $\widetilde{P}(x)$ in Eq. (9) for the domain-size distribution function of the three-state Potts model on the square (solid curve) and the triangular (dashed curve) lattice. The scaling variable is $x=A(t) / \overline{A(t)}$. tions, e.g., the Langevin formalism, computer-simulation calculations on kinetic lattice models such as the Potts models provide insight into the microscopic mechanism of the growth and interface dynamics. The conventional picture of the spinodal-decomposition process involves an evaporation-condensation mechanism. We have investigated this mechanism by calculating the time dependence of isolated Potts spins which are the carriers of the growth process. These isolated Potts spins can be identified on the microconfigurations in Figs. 2 and 3. It is clear from a simple topological reasoning that the twoand three-state Potts models have very different properties as far as these isolated Potts spins are concerned. In both models the isolated Potts spins are generated at the domain interfaces as a consequence of the conservation law. However, in the two-state model isolated Potts spins can only be identified within the domains, whereas in the three-state model isolated Potts spins occur both within the domains as well as in the very domain boundary where Potts spins different from those of the adjacent domains are interfacially adsorbed in the domain boundary. We have conducted a detailed study of the dynamics of isolated Potts spins in the case of the square lattice. As a definition of an isolated Potts spin within a domain on the square lattice, we require that all eight nearestand next-nearest-neighbor spins to the spin under consideration have to be in the same Potts state and different from that of the spin considered. The distinction between the two different types of isolated Potts spins is, of course, somewhat meaningless at very early times and during the nucleation period.

In Figs. 13 and 14 are shown the results for the number of isolated Potts spins, $\rho_{1}(t)$, in the two- and three-state models on the square lattice. The data for the number for the three-state model are separated into its two contributions, $\rho_{1}=\rho_{1}^{\text {domain }}+\rho_{1}^{\text {interface }}$, whereas $\rho_{1}=\rho_{1}^{\text {domain }}$ for the two-state model. It appears from these figures that

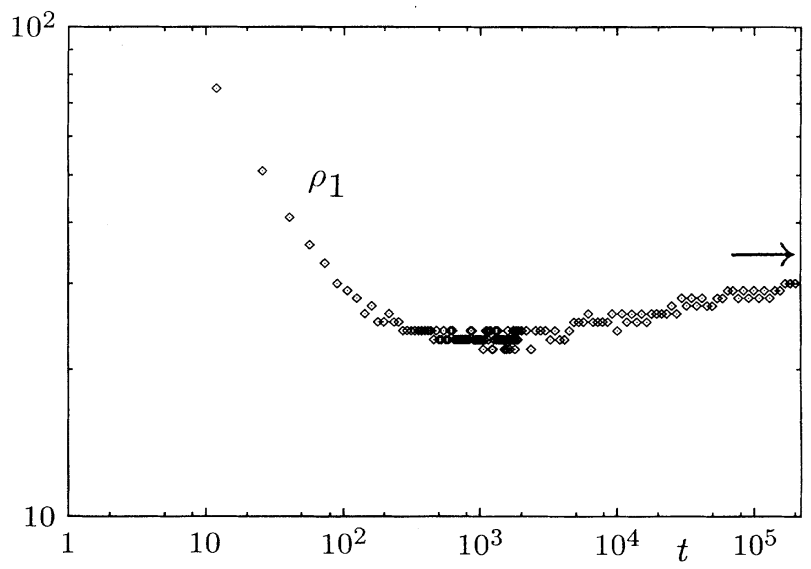

FIG. 13. Time dependence of the number of isolated Potts spins, $\rho_{1}$, within the domains of the two-state Potts model on a square lattice. The equilibrium value of the number of isolated Potts spins in a macroscopic Potts-ordered domain is indicated by an arrow. 


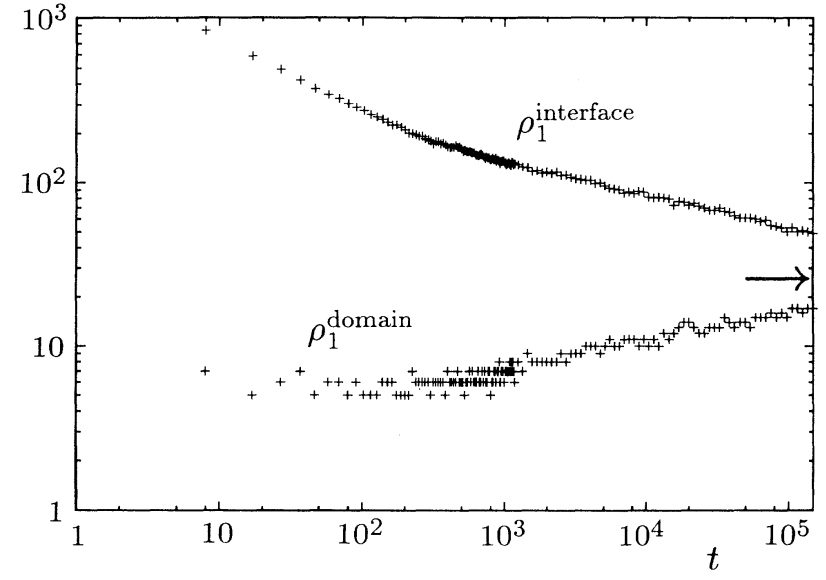

FIG. 14. Time dependence of the number of isolated Potts spins within the domains, $\rho_{1}^{\text {domain }}$, and in the domain boundaries, $\rho_{1}^{\text {interface }}$, of the three-state Potts model on a square lattice. The equilibrium value of the number of isolated Potts spins in a macroscopic Potts-ordered domain is indicated by an arrow.

$\rho_{1}^{\text {domain }}$ for both models decreases at early times, goes through a minimum, and then increases again. For the three-state model, $\rho_{1}^{\text {interface }}$ decreases for all times. The behavior of $\rho_{1}^{\text {domain }}$ reflects that at early stages after the nucleation period, broken Potts bonds mostly occur at the interfaces; i.e., most defects are localized and adsorbed in the domain boundaries. At this stage the inside of the domains is not fully equilibrated and the domain order parameter is larger than the equilibrium bulk Potts order parameter. Hence there is a kind of "overshooting effect" in the order parameter in the intermediate time regime after the quench. At later times the growing domains start equilibrating, which requires solution of "wrong" Potts spins within the domains. These spins are launched from the interface. The isolated Potts spins within the domains are carriers of the late-stage growth process which is only facilitated by long-range diffusion of the isolated Potts spins across the domains. These observations can be used to clarify aspects of the transient growth behavior.

\section{E. Transient growth behavior}

The data presented in Figs. 13 and 14 for the number of isolated Potts spins within the ordered domains provide some insight into the transient growth behavior of spinodal decomposition processes in two- and threecomponent systems.

The transient regime is caused by short-range diffusional processes along the domain-boundary network contrary to the asymptotic growth which is controlled by long-range diffusion of isolated Potts spins across the domains. From the growth data and the analysis of this data in Figs. 4-9, for both the two- and three-state models on both lattices, it is found that at intermediate times the growth behavior is effectively described by an algebraic growth law, Eq. (3) with an exponent $n \sim 0.25$. A simple argument due to Mullins ${ }^{6}$ in fact gives $n=\frac{1}{4}$ for interface diffusion. An early simulation study of the ordering dynamics in conserved multistate Potts models systems also found low exponent values, which can be related to the transient regime. ${ }^{44}$ The higher the number of Potts states, the lower the value of the effective exponent.

It is obvious for topological reasons that this transient regime should be more dominant in the three-component system. In a domain boundary between two domains, the third component can conveniently be adsorbed. The presence of the third component in the boundary enhances the probability for kink formation in the boundary, which in turn facilitates boundary migration mediated by short-range diffusion. This is borne out by direct inspection of microconfigurations such as those in Figs. 2 and 3 and by the time dependence of the density of isolated Potts spins which are the carriers for the growth process (cf. Figs. 13 and 14). In the two-state model, isolated Potts spins are generated at the boundary, but can only exist when the domains where they effectively facilitate transport across the domains. In the three-state model, isolated Potts spins can be present both within the domains and at the domain boundary. The simulations show that the number of isolated Potts spins in the domains only rise to their bulk equilibrium value at a much later time in the three-state model than in the twostate model. This provides a microscopic explanation of the marked difference between the approach to the asymptotic growth behavior of the two- and three-state models, as shown in Figs. 4-9. The transient regime is much more extended in the three-state model. The transient regime also depends on details of the model, in particular the lattice structure. The crossover to asymptotic growth behavior is found to be slower on the triangular lattice than on the square lattice.

\section{DISCUSSION AND SUMMARY: UNIVERSALITY OF ORDERING DYNAMICS IN CONSERVED TWO- AND THREE-COMPONENT SYSTEMS}

The results presented in this paper for the ordering dynamics and spinodal-decomposition processes in two- and three-state Potts models with conserved order parameter suggest that the asymptotic late-state growth law is given by the classical Lifshitz-Slyozov law [Eq. (1)] with exponent value $n=\frac{1}{3}$. The result is independent of the number of components of the order parameter and details such as the underlying lattice structure. Hence the presence of vertices in the domain boundary does not influence the asymptotic growth exponent, although it slows down the overall growth rate. However, the intermediate-stage growth behavior is strongly influenced by transient effects due to diffusion along the interfaces, which leads to lower effective growth exponents as in Eq. (3). The crossover from transient behavior to asymptotic growth is slower the higher the number of conserved order-parameter components, and it is slower on the triangular lattice than on the square lattice.

Our results for the two-state model are consistent with earlier computer-simulation work on the two-component 
Ising model, ${ }^{45}$ as well as with theoretical work on phenomenological field-theoretical models (e.g., the Langevin and Cahn-Hilliard models) (Refs. 29, 31, 32, 34, and 37) and nonlinear coupled-map models. ${ }^{30,40}$ All these studies on rather different models in both two and three spatial dimensions which are subject to the same conservation law for the order parameter lead to the same growth exponent, which suggests a remarkable degree of universality. Our finding in the present work of the same growth law with the same exponent value for the two- and threestate conserved Potts models provides numerical evidence for the existence of a single large universality class which describes ordering dynamics in systems with conserved order parameter, independent of the number of components of the order parameter.

An important characteristic of the universality class is the dynamical scaling functions describing the various properties of the system. In earlier numerical studies of spinodal-decomposition processes in two-component systems, the dynamical scaling properties of the ordering process were established via calculations of the timedependent static structure function ${ }^{31,43,45}$ and paircorrelation function. ${ }^{31,37}$ In the present work, we calculated the domain-size distribution function for the threestate model on two different lattices and found (cf. Fig. 12) that the ordering process obeys dynamical scaling at late stages and that the scaling function for the domainsize distribution function, within the numerical accuracy, is independent of lattice structure. This result gives further testimony to universality.

The available experimental data for spinodal decomposition processes, e.g., in alloys, polymer blends, fluid mix- tures, and ceramics (for a review, see, e.g., Binder ${ }^{2}$ ), provides further evidence for the Lifshitz-Slyozov growth law, although under many practical circumstances for spinodal decomposition, e.g., in metallic alloys, ${ }^{2}$ the transient behavior is prevailing and the asymptotic regime is not reached within the time available. Quite often the finding of a transient growth law as in Eq. (2) is taken as evidence for spinodal decomposition. ${ }^{2}$ Transient behavior becomes more severe when more components are involved; e.g., in experimental studies of spinodal decomposition in ternary alloys it is found that the effective growth exponent is often lower than for binary alloys. ${ }^{17}$ These findings are consistent with the results of the present model study.

The suggestion of the existence of a single large universality class for conserved systems undergoing spinodal decomposition, together with the well-accepted theory for a large universality class for ordering dynamics in systems with nonconserved order parameter, ${ }^{3,11}$ implies that universality in ordering dynamics is a much more general phenomenon than in static and dynamic critical phenomena ${ }^{60}$ where both spatial dimensionality and the number of components of the order parameter are relevant variables.

\section{ACKNOWLEDGMENTS}

This work was supported by the Danish Natural Science Research Council under Grant No. 11-7785, by the Danish Research Academy, and by the Danish National Computer Center UNI.C. Access to the SUN cluster at the PICL-LAB at UCLA is gratefully acknowledged.
${ }^{1}$ J. S. Langer, Ann. Phys. (N.Y.) 65, 53 (1991).

${ }^{2} \mathrm{~K}$. Binder, in Phase Transformations in Materials, edited by $\mathbf{P}$. Haasen, Materials Science and Technology, Vol. 5 (VCH, Weinheim, Germany, 1990), p. 405.

${ }^{3}$ J. D. Gunton, M. San Miguel, and P. S. Sahni, in Phase Transitions and Critical Phenomena, edited by C. Domb and J. L. Lebowitz (Academic, New York, 1983), Vol. 8, p. 267.

${ }^{4}$ I. M. Lifshitz and V. V. Slyozov, J. Phys. Chem. Solids 19, 35 (1961).

${ }^{5}$ A. Sadiq and K. Binder, J. Stat. Phys. 35, 517 (1984).

${ }^{6} \mathrm{~W}$. W. Mullins, in Metal Surfaces (American Society for Metals, Metals Park, OH, 1962), Vol. 17.

${ }^{7}$ O. G. Mouritsen, P. J. Shah, and J. V. Andersen, Phys. Rev. B 42, 4506 (1990).

${ }^{8}$ O. G. Mouritsen, P. J. Shah, J. V. Andersen, H. F. Poulsen, and H. Bohr, Phys. Scr. T 38, 55 (1991).

${ }^{9}$ H. F. Poulsen, J. V. Andersen, N. H. Andersen, H. Bohr, and O. G. Mouritsen, Phys. Rev. Lett. 66, 465 (1991).

${ }^{10}$ W. W. Mullins and J. Viñals, Acta Metall. 37, 991 (1989).

${ }^{11} \mathrm{O}$. G. Mouritsen, in Kinetics of Ordering and Growth at Surfaces, edited by M. G. Lagally (Plenum, New York, 1990), p. 1.

${ }^{12}$ For an experimental overview of the field of ordering dynamics, see the selection of papers in Dynamics of Ordering Processes in Condensed Matter, edited by $\mathbf{S}$. Komura and $\mathrm{H}$. Furukawa (Plenum, New York, 1988).
${ }^{13}$ S. Katano and M. Iizumi, Phys. Rev. Lett. 52, 835 (1984).

${ }^{14}$ B. D. Gaulin, S. Spooner, and Y. Morii, Phys. Rev. Lett. 59, 668 (1987).

${ }^{15}$ S. Komura, K. Osamura, H. Fujii, and T. Takeda, Phys. Rev. B 31, 1278 (1985).

${ }^{16}$ P. Wilzius, F. S. Bates, and W. R. Heffneer, Phys. Rev. Lett. 60, 1538 (1988).

${ }^{17} \mathbf{P}$. Guyot and J. P. Simon, in Dynamics of Ordering Processes in Condensed Matter (Ref. 12), p. 211.

${ }^{18}$ P. W. Voorhees, J. Stat. Phys. 38, 231 (1985).

${ }^{19}$ H. Tomita, Prog. Teor. Phys. 75, 482 (1986).

${ }^{20}$ H. Furukawa, Adv. Phys. 34, 703 (1985).

${ }^{21}$ D. A. Huse, Phys. Rev. B 34, 7845 (1986).

${ }^{22}$ G. F. Mazenko, O. T. Valls, and F. C. Zhang, Phys. Rev. B 32, 5807 (1985).

${ }^{23}$ Q. Zheng and J. D. Gunton, Phys. Rev. A 39, 4848 (1989).

${ }^{24}$ A. J. Bray, Phys. Rev. B 41, 6724 (1990).

${ }^{25}$ G. F. Mazenko, Phys. Rev. Lett. 63, 1605 (1989).

${ }^{26}$ G. F. Mazenko, Phys. Rev. B 42, 4487 (1990).

${ }^{27}$ K. R. Elder, B. Morin, M. Grant, and R. C. Desai, Phys. Rev. B 44, 6673 (1991).

${ }^{28}$ M. San Miguel, J. D. Gunton, G. Dee, and P. Sahni, Phys. Rev. B 23, 2334 (1981).

${ }^{29}$ R. Toral, A. Chakrabarti, and J. D. Gunton, Phys. Rev. Lett. 60, 2311 (1988).

${ }^{30}$ Y. Oono and S. Puri, Phys. Rev. A 38, 434 (1988); S. Puri and 
Y. Oono, ibid. 38, 1542 (1988).

${ }^{31}$ T. M. Rogers, K. R. Elder, and R. C. Desai, Phys. Rev. B 37, 9638 (1988).

${ }^{32}$ O. T. Valls and G. F. Mazenko, Phys. Rev. B 38, 11650 (1988).

${ }^{33}$ K. R. Elder, T. M. Rogers, and R. C. Desai, Phys. Rev. B 38, 4725 (1988).

${ }^{34}$ A. Milchev, D. W. Heermann, and K. Binder, Acta Metall. 36, 377 (1988).

${ }^{35}$ J. Viñals and D. Jasnow, Phys. Rev. B 37, 9582 (1988).

${ }^{36}$ T. M. Rogers and R. C. Desai, Phys. Rev. B 39, 11956 (1989).

${ }^{37}$ E. T. Gawlinski, J. D. Gunton, and J. Viñals, Phys. Rev. B 39, 7266 (1989).

${ }^{38}$ A. Chakrabarti, R. Toral, J. D. Gunton, and M. Muthukumar, Phys. Rev. Lett. 63, 2072 (1989).

${ }^{39}$ A. Onuki and H. Nishimori, Phys. Rev. B 43, 13649 (1991).

${ }^{40}$ S. Puri, R. C. Desai, and R. Kapral, Physica D 50, 207 (1991).

${ }^{41}$ A. Chakrabarti, Phys. Rev. B 45, 9620 (1992).

${ }^{42 J}$. Viñals, M. Grant, M. San Miguel, J. D. Gunton, and E. T. Gawlinski, Phys. Rev. Lett. 54, 1264 (1985).

${ }^{43}$ C. Roland and M. Grant, Phys. Rev. B 39, 11971 (1989).

${ }^{44}$ G. S. Grest and P. S. Sahni, Phys. Rev. B 30, 226 (1984).

${ }^{45}$ J. G. Amar, F. E. Sullivan, and R. D. Mountain, Phys. Rev. B
37, 196 (1988)

${ }^{46}$ M. C. Tringides, Phys. Lett. 133, 325 (1988).

${ }^{47}$ J. G. Amar and F. Sullivan, Comput. Phys. Commun. 55, 287 (1989).

${ }^{48}$ W. Schleier, G. Besold, and K. Heinz, J. Stat. Phys. 66, 1091 (1992).

${ }^{49}$ J. F. Annett and J. R. Banavar, Phys. Rev. Lett. 68, 2941 (1992).

${ }^{50}$ A. Chakrabarti, J. B. Collins, and J. D. Gunton, Phys. Rev. B 38, 6894 (1988).

${ }^{51}$ C. Jeppesen and O. G. Mouritsen (unpublished).

${ }^{52}$ F. Y. Wu, Rev. Mod. Phys. 54, 2235 (1982).

${ }^{53}$ T. Schultz, D. Mattis, and E. Lieb, Rev. Mod. Phys. 36, 856 (1964).

${ }^{54}$ R. M. Houtappel, Physica 16, 425 (1950).

${ }^{55}$ K. Binder and D. Stauffer, Phys. Rev. Lett. 33, 1006 (1974).

${ }^{56}$ P. S. Sahni, D. J. Srolovitz, G. S. Grest, M. P. Anderson, and S. A. Safran, Phys. Rev. B 28, 2705 (1983).

${ }^{57}$ P. J. Shah and O. G. Mouritsen, Phys. Rev. B 41, 7003 (1990).

${ }^{58}$ E. Vives and A. Planes, Phys. Rev. B 47, 2557 (1993).

${ }^{59}$ C. Yeung, Phys. Rev. B 39, 9652 (1989).

${ }^{60}$ P. C. Hohenberg and B. I. Halperin, Rev. Mod. Phys. 49, 435 (1977). 